DAMTP-2004-84

hep-th/0408244v2

\title{
Quantum, higher-spin, local charges in symmetric space sigma models
}

\author{
J.M. Evans ${ }^{a}$, D. Kagan ${ }^{a}$, N.J. MacKay ${ }^{b}$, C.A.S. Young ${ }^{a}$, \\ ${ }^{a}$ DAMTP, Centre for Mathematical Sciences, University of Cambridge, \\ Wilberforce Road, Cambridge CB3 OWA, UK \\ ${ }^{b}$ Department of Mathematics, University of York, \\ Heslington Lane, York YO10 5DD, UK \\ E-mail: J.M.Evans@damtp.cam.ac.uk, D.Kagan@damtp.cam.ac.uk, \\ nm15@york.ac.uk, C.A.S.Young@damtp.cam.ac.uk
}

\begin{abstract}
Potential anomalies are analysed for the local spin-3 and spin-4 classically conserved currents in any two-dimensional sigma model on a compact symmetric space $G / H$, with $G$ and $H$ classical groups. Quantum local conserved charges are shown to exist in exactly those models which also possess quantum non-local (Yangian) charges. The possibility of larger sets of quantum local charges is discussed and shown to be consistent with known S-matrix results and the behaviour of the corresponding Yangian representations.
\end{abstract}

\section{Introduction}

Sigma models in two spacetime dimensions are known to be quantum integrable if their target manifolds are compact Lie groups or certain other symmetric spaces [1]-[8], namely

$$
\begin{gathered}
S O(n+1) / S O(n), \quad S U(n) / S O(n), \quad S U(2 n) / S p(n), \\
S O(2 n) / S O(n) \times S O(n), \quad S p(2 n) / S p(n) \times S p(n),
\end{gathered}
$$

together with a finite number of examples involving exceptional groups. It is a longstanding result 22 that a sigma model on a symmetric space $G / H$ with $H$ simple $^{1}$ possesses a conserved non-local charge at the quantum level, which implies integrability. The real and symplectic Grassmannians in (11) have $H$ non-simple, however, and are comparatively recent additions to this list of integrable models. S-matrices for these new cases were proposed and tested in [3, 4, 5] and it was subsequently shown [6] that they too possess quantum non-local charges.

\footnotetext{
${ }^{1}$ Here, and throughout this paper, we shall use 'simple' to mean that the corresponding Lie algebra has no non-trivial ideals. Hence $U(1)$ is simple in our terminology, in addition to the usual non-abelian simple groups of the Cartan-Killing classification $[9]$.
} 
An alternative criterion for integrability, either classical or quantum, is the existence of higher-spin, local conserved charges (see e.g. 7, 8, 10, 11, 12, 13]). These offer an independent check on whether the list in (11) is now correct and complete, which is particularly important in view of the fact that two families were overlooked until recently. In this paper we give a uniform treatment of sigma models on (irreducible [9]) symmetric spaces $G / H$ where $G$ (simple) and $H$ are compact classical groups: we investigate the quantum behaviour of all local conserved currents of spin three or four using an approach due to Goldschmidt and Witten [8] which is based purely on symmetry arguments. ${ }^{2}$

We discuss general aspects of the classical symmetric space sigma models in section 2, before outlining how the analysis of the local currents is carried out, and also stating our results, in section 3. Sections 4 and 5 provide the case-by-case details of the analysis on which the results rest. Our findings are in complete agreement with the studies of non-local charges cited above. In addition to the basic question of integrability of the models, however, there are also very interesting consequences which follow from the coexistence of local and non-local conserved quantities. Both constrain the S-matrix in powerful ways (beyond factorisation) and the consistency of these constraints is highly non-trivial [15, 14, 17, 11. We discuss, in section 6, how the existence of quantum local charges fits with the properties of the Yangian quantum groups generated by the non-local charges (see e.g. [18, 19, 20, 21]).

\section{General aspects of the models}

One way to formulate the sigma model with target space $G / H$ is to introduce a field $g\left(x^{\mu}\right) \in G$ which transforms under both a global symmetry $g\left(x^{\mu}\right) \mapsto U g\left(x^{\mu}\right)$, for $U \in G$, and also under local gauge transformations $g\left(x^{\mu}\right) \mapsto g\left(x^{\mu}\right) h\left(x^{\mu}\right)$ for $h\left(x^{\mu}\right) \in H$, thereby ensuring that physical degrees of freedom are properly confined to the coset space. ${ }^{3}$ An alternative approach, which we shall adopt, is to construct the model without any gauge redundancy. To do this we use the fact that each symmetric space can be parametrised by a particular set of unitary matrices, so there is a field $\Phi_{a b}\left(x^{\mu}\right)$ obeying $\Phi \Phi^{\dagger}=1$ as well as certain additional constraints. We will describe the precise nature of these constraints for each family of classical symmetric spaces in sections 4 and 5 ; in this section we will concentrate on features common to all the models.

We must specify how $\Phi$ transforms under $G$, and we will distinguish two possibilities:

$$
\text { (a) } \Phi \mapsto U \Phi U^{\dagger} \quad \text { or } \quad \text { (b) } \Phi \mapsto U \Phi U^{T}
$$

where $U \in G$. The constraints on $\Phi$ ensure that it can be related to a canonical form, by

\footnotetext{
${ }^{2}$ Goldschmidt and Witten [8] established quantum integrability for sigma models on spheres and classical Lie groups (see also [1]); the method was also used in 6], but only for a rather special set of currents, to confirm the integrability of the $S O(2 n) / S O(n) \times S O(n)$ models (see section 5 ).

${ }^{3}$ All groups are classical and elements of them are unitary matrices in the defining representations; similarly, elements of the corresponding Lie algebras are traceless antihermitian matrices.
} 
which we mean some fixed, unitary matrix $N$, as follows:

$$
\text { (a) } \Phi=g N g^{\dagger} \quad \text { or } \quad \text { (b) } \Phi=g N g^{T} \text {. }
$$

Any given $\Phi\left(x^{\mu}\right)$ thus corresponds to (many) $g\left(x^{\mu}\right)$, and this is exactly the field introduced in the coset description. The correspondence between the two approaches is completed by identifying $H$ as the subgroup which preserves $N$ :

$$
\text { (a) } h N h^{\dagger}=N \quad \text { or } \quad \text { (b) } h N h^{T}=N \text {. }
$$

Note that cases (a) and (b) coincide if $G$ is an orthogonal group. Case (b) applies to the families $S U(n) / S O(n)$ and $S U(2 n) / S p(n)$, while case (a) applies to all other classical symmetric spaces. For example, if $\Phi$ is a traceless, hermitian, $2 n \times 2 n$ matrix transforming under $G=S U(2 n)$ as in (2a), then (3a) certainly holds with $N=\operatorname{diag}(1, \ldots, 1,-1, \ldots,-1)$ and (4a) implies that $H=S(U(n) \times U(n))$. In this way we recover one of the complex Grassmannians. On the other hand, the coset $S U(n) / S O(n)$ is obtained by taking $\Phi$ to be a complex, symmetric, $n \times n$ matrix with $\operatorname{det} \Phi=1$. It transforms under $S U(n)$ according to (2b), so (3) holds with $N$ the identity matrix and the subgroup $H$ is indeed $S O(n)$.

Underlying this construction, in general, is the Cartan immersion $G / H \rightarrow G$ [20, 22]. But the validity of the approach can be checked case by case when $G$ and $H$ are classical, using basic results from linear algebra, as in the examples above (and as in [3, 4, 23]). When we consider the various families of symmetric spaces in more detail in sections 4 and 5 , we will simply state the properties required of $\Phi$ and specify the canonical form $N$.

The lagrangian for the $G / H$ sigma model, with field $\Phi\left(x^{\mu}\right)$ is $\operatorname{Tr}\left(\partial_{\mu} \Phi \partial^{\mu} \Phi^{\dagger}\right)$ supplemented by the relevant constraints, among them $\Phi \Phi^{\dagger}=1$ (these can be enforced by Lagrange multipliers). The theory is invariant under $G$, with Noether current

$$
j_{\mu}=\frac{1}{2} \Phi \partial_{\mu} \Phi^{\dagger} \in \mathfrak{g}
$$

where $\mathfrak{g}$ is the Lie algebra of $G$. This current transforms

$$
j_{\mu} \mapsto U j_{\mu} U^{\dagger}
$$

under (2) in cases (a) and (b). The equations of motion for the model are ${ }^{4}$

$$
\partial_{-} j_{+}=-\partial_{+} j_{-}=\left[j_{+}, j_{-}\right]
$$

or in terms of the fields,

$$
\partial_{+} \partial_{-} \Phi=2\left\{j_{+}, j_{-}\right\} \Phi .
$$

Note that this is independent of the details of the constraints.

\footnotetext{
${ }^{4}$ Orthonormal and light-cone components of spacetime vectors are related by $u_{ \pm}=u_{0} \pm u_{1}$.
} 
The form of equation (17) is responsible for the classical integrability of the symmetric space sigma models. It allows us to construct local conserved currents of spin $m$ :

$$
\partial_{-} \operatorname{Tr}\left(j_{+}^{m}\right)=0
$$

(and similarly with \pm interchanged). It is, of course, essential to know when such currents are non-zero, and this leads us to the question of invariants on symmetric spaces.

Let $\mathfrak{h}$ be the Lie algebra of $H$ and $\mathfrak{m}$ its orthogonal complement in $\mathfrak{g}$; then

$$
\mathfrak{g}=\mathfrak{h} \oplus \mathfrak{m} \quad \text { with } \quad[\mathfrak{h}, \mathfrak{h}] \subset \mathfrak{h}, \quad[\mathfrak{h}, \mathfrak{m}] \subset \mathfrak{m}, \quad[\mathfrak{m}, \mathfrak{m}] \subset \mathfrak{h}
$$

It follows from the definition of $H$ in $(4 a)$ or $(4 b)$ that $\mathfrak{h}$ and $\mathfrak{m}$ are the \pm 1 eigenspaces of the following map on $\mathfrak{g}$

$$
\text { (a) } \sigma: X \mapsto N X N^{\dagger} \quad \text { or } \quad \text { (b) } \sigma: X \mapsto N X^{*} N^{\dagger} \text {. }
$$

In other words, $\sigma$ is the involutive automorphism of $\mathfrak{g}$ which defines the symmetric space.

Now although $j_{\mu}$ belongs to $\mathfrak{g}$, it is conjugate to something in the subspace $\mathfrak{m}$, specifically

$$
j_{\mu}=-g k_{\mu} g^{-1} \quad \text { where } \quad k_{\mu}=\frac{1}{2}\left(g^{-1} \partial_{\mu} g-\sigma\left(g^{-1} \partial_{\mu} g\right)\right) \in \mathfrak{m}
$$

which follows on substituting (3) in (15). In considering $\operatorname{Tr}\left(j_{+}^{m}\right)=(-1)^{m} \operatorname{Tr}\left(k_{+}^{m}\right)$ we are therefore concerned not merely with symmetric $G$-invariant tensors on $\mathfrak{g}$, but actually with symmetric $H$-invariant tensors on $\mathfrak{m}$. We shall refer simply to invariants on $G / H$ from now on. An analysis of these symmetric $G / H$ invariants, including the question of which of them are primitive, meaning that they are not combinations of invariants of lower order, has been given in [12] (see also [13]) in the context of classical conserved charges; we shall have frequent recourse to these results.

Discrete symmetries of the $G / H$ sigma models also play a very important role. All models are invariant under

$$
\Phi \mapsto \Phi^{*}, \quad j_{\mu} \mapsto j_{\mu}^{*}=-j_{\mu}^{T} .
$$

Even if this symmetry is trivial, in that $\Phi$ is real, the resulting antisymmetry of the current has important consequences, e.g. for the vanishing of certain trace invariants. Other discrete symmetries will be discussed as they arise.

\section{Overview of anomaly counting and statement of results}

Suppose that in some model there are $n_{C}$ independent, local quantities $C_{k}$ which obey classical conservation equations $\partial_{-} C_{k}=0$ and which have identical behaviour under all symmetries, continuous and discrete. The Goldschmidt-Witten (GW) approach [8] to investigating the effect of quantization is to count the number of independent, local terms $A_{i}$ with the correct symmetries to appear as quantum modifications of these equations, making free use of the classical equations of motion, and to count similarly the number of 
independent terms $B_{j}$ which have the correct symmetries and which are derivatives of local expressions. Let the number of these terms be $n_{A}$ and $n_{B}$ respectively. If $n_{C}>n_{A}-n_{B}$ then there is at least one combination of conservation equations which survives quantization, because its quantum modification is a derivative. When this occurs for a given class of conservation equations (characterised by their symmetries, including spin) we will say ' $\mathrm{GW}$ works', and if not, 'GW fails'. Note, however, that 'GW fails' does not imply that there is no quantum conservation equation, only that we can draw no definite conclusion from the counting.

We will investigate the quantum modifications to (9) with $m=2,3,4$. The anomalies ( $A$ 's) and derivatives ( $B$ 's) must be $G$-invariant and so can be expressed in terms of ${ }^{5}$
(a) $\operatorname{Tr}\left(\mathcal{D}_{1} \Phi \mathcal{D}_{2} \Phi \ldots \mathcal{D}_{r} \Phi\right) \quad$ or
(b) $\operatorname{Tr}\left(\mathcal{D}_{1} \Phi \mathcal{D}_{2} \Phi^{\dagger} \ldots \mathcal{D}_{2 s-1} \Phi \mathcal{D}_{2 s} \Phi^{\dagger}\right)$

depending on the transformation in (2), where each $\mathcal{D}_{i}$ is a product of powers of $\partial_{+}$and $\partial_{-}$, or the identity operator. But it follows from $\Phi \Phi^{\dagger}=1$ and consequences of it such as $\left(\partial_{\mu} \Phi\right) \Phi^{\dagger}=-\Phi\left(\partial_{\mu} \Phi\right)^{\dagger}$ that all derivatives of $\Phi$ can be re-expressed in terms of the Noether current $j_{\mu}$, its derivatives, and $\Phi$ fields without derivatives. Moreover, any quantity of the form (14b), or (14a) with $r$ even, can be written entirely in terms of $j_{\mu}$ and its derivatives, while any quantity (14 $)$ with $r$ odd can be written in terms of $j_{\mu}$, its derivatives, and a single $\Phi$. Finally, we can use (17) to eliminate any occurrences of $\partial_{-} j_{+}$or $\partial_{+} j_{-}$.

The simplest application of GW counting is to energy-momentum conservation. The classical equation $\partial_{-} \operatorname{Tr}\left(j_{+}^{2}\right)=0$ will be modified in the quantum theory (scale-invariance is broken) but we can check, in each model (see sections 4 and 5), that the only relevant anomaly terms with the correct symmetries are those involving just $j_{\mu}$ and its derivatives. The quantum modification must be even under (13) and so must be proportional to $\operatorname{Tr}\left(j_{-} \partial_{+} j_{+}\right)=\partial_{+} \operatorname{Tr}\left(j_{-} j_{+}\right)$, on using the equations of motion. Thus energy-momentum is conserved quantum-mechanically, as expected.

Consider next the classical spin-3 current, $C=\operatorname{Tr}\left(j_{+}^{3}\right)$, which is odd under (13). For this to be non-vanishing, the symmetric space must have a 3rd-order invariant, which occurs only for $S U(n) / S O(n)$ and $S U(2 n) / S p(n)$ with $n \geq 3$ [12. But in these families the field $\Phi$ transforms as in (2b), so $G$-invariants are of type (14b) and, as argued above, anomalies and derivatives can once again be written entirely in terms of $j_{\mu}$ and its derivatives. The only terms with the correct symmetries, changing sign under (13), are

$$
A=\operatorname{Tr}\left(j_{-}\left\{j_{+}, \partial_{+} j_{+}\right\}\right), \quad B=\partial_{+} \operatorname{Tr}\left(j_{-} j_{+}^{2}\right)
$$

Hence GW works (in fact $A=B$ ) and the spin-3 current survives quantization.

\footnotetext{
${ }^{5}$ When $G$ is $S O(n)$ or $S U(n)$ we can also construct invariants using $\varepsilon$ tensors. These are never relevant for the anomalies and derivatives associated with currents (9), however, because the $\varepsilon$ invariants either reduce to traces or else can be distinguished from trace-type invariants by symmetries. Some details are given in sections 4 and 5 .
} 
Now consider the conserved quantities of spin-4:

$$
C_{1}=\left(\operatorname{Tr}\left(j_{+}^{2}\right)\right)^{2}, \quad C_{2}=\operatorname{Tr}\left(j_{+}^{4}\right),
$$

which are both invariant under (13). These are distinct if there is a primitive symmetric 4th-order invariant on $G / H$; if there is not, $C_{2}$ is proportional to $C_{1}$. Thus $n_{C}=1$ or 2 , the number of independent symmetric 4th-order invariants on $G / H$.

Before listing possible anomalies and derivatives for the spin-4 case it is helpful to introduce some new notation by defining:

$$
j_{++}=\partial_{+} j_{+}, \quad j_{+++}=\partial_{+} j_{++}+\left[j_{+}, j_{++}\right], \quad j_{++++}=\partial_{+} j_{+++}+\left[j_{+}, j_{+++}\right] .
$$

The advantage of working with these modified derivatives, all of which clearly belong to $\mathfrak{g}$, is that they each obey $j=-g k g^{-1}$ for some $k \in \mathfrak{m}$, generalising (12). This, and properties which follow from it, will prove very convenient. Note also $j \mapsto j^{*}=-j^{T}$ under (13).

The counting of anomalies and derivatives is much more intricate for the spin- 4 case. Let us first write down all possible anomaly terms which are constructed from $j_{\mu}$ alone and which are invariant under (13). There are five independent terms in general:

$$
\begin{aligned}
& A_{1}=\operatorname{Tr}\left(j_{-} j_{++++}\right) \\
& A_{2}=\operatorname{Tr}\left(j_{-} j_{+}\right) \operatorname{Tr}\left(j_{+} j_{++}\right) \\
& A_{3}=\operatorname{Tr}\left(j_{-} j_{++}\right) \operatorname{Tr}\left(j_{+}^{2}\right) \\
& A_{4}=\operatorname{Tr}\left(j_{+} j_{-} j_{+} j_{++}\right) \\
& A_{5}=\operatorname{Tr}\left(j_{+}^{2}\left\{j_{-}, j_{++}\right\}\right)
\end{aligned}
$$

Other possibilities which involve $\Phi$ fields and which we encounter in some models are:

$$
\begin{aligned}
& A_{6}=\operatorname{Tr}\left(\Phi j_{-} j_{+}\right) \operatorname{Tr}\left(\Phi j_{+} j_{++}\right) \\
& A_{7}=\operatorname{Tr}\left(\Phi j_{+}^{2}\left\{j_{-}, j_{++}\right\}\right)
\end{aligned}
$$

The derivative terms which involve $j_{\mu}$ alone and which have the correct symmetries are:

$$
\begin{aligned}
& B_{1}=\partial_{+} \operatorname{Tr}\left(j_{-} j_{+++}\right) \\
& B_{2}=\partial_{-} \operatorname{Tr}\left(j_{++}^{2}\right) \\
& B_{3}=\partial_{+}\left(\operatorname{Tr}\left(j_{-} j_{+}\right) \operatorname{Tr}\left(j_{+}^{2}\right)\right) \\
& B_{4}=\partial_{+} \operatorname{Tr}\left(j_{-} j_{+}^{3}\right)
\end{aligned}
$$

It will turn out that these are the only derivative terms that are relevant. (Note that $\partial_{-} \operatorname{Tr}\left(j_{+} j_{+++}\right)$is proportional to $B_{2}$, using the equations of motion.)

It is clear that $B_{1}, B_{2}, B_{3}$ are always independent, but $B_{4}$ is an additional independent quantity iff there is a primitive 4 th-order invariant on $G / H$, otherwise it is proportional to $B_{3}$. Thus

$$
n_{C}=1 \Rightarrow n_{B}=3, \quad n_{C}=2 \Rightarrow n_{B}=4
$$


The question of how the various anomaly terms are related when $n_{C}=1$ is more subtle for two reasons. First, the lack of a primitive 4th-order invariant implies only that totally symmetrized traces reduce to lower-order invariants. For instance, the appropriate combination of $A_{4}$ and $A_{5}$ in (18) must reduce in these circumstances, but they need not be individually reducible. Second, $G$-invariant quantities simply work differently if they involve $\Phi$, such as $A_{6}$ and $A_{7}$ in (19), because, unlike the currents, $\Phi$ does not belong to $\mathfrak{g}$. These cases require special consideration (one approach is explained in the appendix).

We have kept the presentation as general as possible for as long as possible; to go further we must state explicitly the constraints obeyed by $\Phi$, the discrete symmetries of the sigma model, and so on. We provide these details in the following sections, 4 and 5, and we specify exactly which anomaly and derivative terms are allowed and independent for each classical symmetric space. In the interests of clarity, however, we state, in advance, the conclusions which follow from GW counting:

(i) All sigma models in (1) possess at least one higher-spin quantum conserved charge.

(ii) GW fails for sigma models on those classical symmetric spaces $G / H$ not in (1).

These broad conclusions provide independent confirmation of the quantum integrability of the models in (1) and also lend support to the suggestion that this list is complete. At a finer level of detail, however, we find:

(iii) The sigma models on $S U(n) / S O(n)$ and $S U(2 n) / S p(n)$ possess a spin-3 quantum conserved current for $n \geq 3$ (these are the classical symmetric spaces with a 3rd-order invariant).

(iv) All sigma models in (1) possess a spin-4 quantum conserved current, with the possible exceptions of $S U(3) / S O(3)$ and $S U(6) / S p(3)$, for which $G W$ fails.

We will also summarise, and clarify in one small respect, the status of local charges in principal chiral models (PCMs) with target spaces simple classical Lie groups $G$ [8, 11]:

(v) There is a quantum spin-3 conserved current when $G$ is $S U(n)$ for $n \geq 3$ (these are the classical groups with a 3rd-order invariant); and there is a quantum spin-4 conserved current for all $G$, with the possible exception of $S U(3)$, for which $G W$ fails.

The analysis of spin-4 currents in PCMs was given in [8, 11] for classical groups $G$ which possess a primitive 4th-order invariant. Of the few classical groups which lack such an invariant, the case $S U(2)=S^{3}$ was also dealt with in [8], as one of the family of sigma models on spheres, and $S O(3)$ is similar. This leaves just the case of $S U(3)$ to consider, which is easily done (see section 5 ).

It is striking that there are just three examples - in (iv) and (v) - where the sigma model is integrable yet GW fails to show the quantum conservation of a spin-4 current (even though it shows the existence of a quantum spin-3 current). We shall return to this point in section 6 . 


\section{Details for the Grassmannian models}

The real, complex and quaternionic (or symplectic) Grassmannians:

$$
S O(p+q) / S O(p) \times S O(q), \quad S U(p+q) / S(U(p) \times U(q)), \quad S p(p+q) / S p(p) \times S p(q)
$$

are each parametrised by a hermitian matrix $\Phi$ and (2h, 3 h 4 a $)$ hold. In the real and complex cases $\Phi$ is $(p+q) \times(p+q)$ with $^{6}$

$$
\Phi^{\dagger}=\Phi, \quad \Phi^{2}=1, \quad \operatorname{Tr} \Phi=p-q, \quad N=\left(\begin{array}{cc}
1_{p \times p} & 0 \\
0 & -1_{q \times q}
\end{array}\right) .
$$

In the quaternionic case $\Phi$ and $N$ are doubled in size, with $\operatorname{Tr} \Phi=2(p-q)$. For the real and quaternionic families $\Phi$ also satisfies reality conditions

$$
\Phi^{*}=\Phi \quad \text { or } \quad \Phi^{*}=J \Phi J^{-1},
$$

respectively, where $J$ is a $2(p+q) \times 2(p+q)$ symplectic structure (a real antisymmetric matrix with $J^{2}=-1$ ). All three families of Grassmannians have a special feature which arises iff $p=q$, namely, a discrete symmetry $\tau: \Phi \mapsto-\Phi, j \mapsto j$, where $j$ is any of the currents $j_{ \pm}$or $j_{+\ldots+}$. (Note that if $p \neq q$ this map does not respect the condition on $\operatorname{Tr} \Phi$.) The conserved quantities (91) are clearly invariant under $\tau$. This symmetry was crucial in showing the existence of quantum conserved non-local charges for the special families of Grassmannians in (11) [6] and it will play an equally important role here.

Using $\Phi=\Phi^{\dagger}$ and the definitions (5) and (17) we find, for any current $j$,

$$
\Phi j=-j \Phi \quad \text { or } \quad \Phi j \Phi^{-1}=-j,
$$

and this has useful consequences: (i) The trace of any odd number of currents vanishes, whether or not it contains a factor of $\Phi$. (ii) $\operatorname{Tr}\left(\Phi j^{2}\right)=0$ for any $j$, from cyclicity of the trace. (iii) $\operatorname{Tr}\left(\Phi j j^{\prime}\right)$ is odd under (13); in the real and symplectic cases (23) then implies that it actually vanishes; in the complex case it need not vanish if $j \neq j^{\prime}$, but we must multiply two such expressions to obtain something even under (13), an example being $A_{6}$ in (19). (iv) Similar restrictions can be derived for traces of $\Phi$ with four currents; thus $A_{7}$ in (19) is non-zero and even under (13), whereas other candidates for anomalies and derivatives, such as $\operatorname{Tr}\left(\Phi j_{+} j_{-} j_{+} j_{++}\right)$and $\partial_{\mp} \operatorname{Tr}\left(\Phi j_{ \pm} j_{+}^{3}\right)$, either vanish or are odd under (13).

These remarks justify the assumption made in section 3 (of using only $j$ 's, no $\Phi$ 's) when establishing quantum conservation of energy-momentum. They also imply that for spin-4 currents, the possible anomalies are limited to $A_{i}$ in (18) and (19) while the only derivatives with the correct symmetries are $B_{j}$ in (201). We now complete the treatment of the spin-4

\footnotetext{
${ }^{6}$ There is a subtlety in the real case: the matrix $\Phi$ actually parametrises $O(p+q) / O(p) \times O(q)=$ $S O(p+q) / S(O(p) \times O(q))$ rather than $S O(p+q) / S O(p) \times S O(q)$ and so it does not quite provide a 'faithful representation' of the latter symmetric space. The relationship between these spaces is analogous to that between $S U(2)$ and $S O(3)$ (which is actually the case $p=3, q=1$ ).
} 
case for each family. (Some additional comments, mainly of use in confirming the counting in the $q=1$ cases, are relegated to an appendix.)

Spin-4 for real Grassmannians

- If $p \geq q \geq 2$ then $n_{C}=2$ and $n_{B}=4$. The possible anomalies are $A_{i}$ with $1 \leq i \leq 5$ and $A_{7}$ if $p \neq q$. If $p=q$, however, then $A_{7}$ is ruled out by $\tau$. Thus for $p \neq q$ we have $n_{A}=6$, $n_{B}=4$ and GW fails; but for $p=q$ we have $n_{A}=5, n_{B}=4$ and GW works.

- If $p>q=1$, the target manifolds are spheres $S^{p}$. There is no primitive 4th-order invariant so $n_{C}=1$ and $n_{B}=3$. But $A_{4}, A_{5}$ and $A_{7}$ can now each be expressed in terms of $A_{2}$ and $A_{3}$. Hence $n_{A}=n_{B}=3$ and GW works for all $p$.

We have not considered invariants involving $\varepsilon$ tensors above, because the real Grassmannians possess a symmetry $\mu: \Phi \mapsto M \Phi M^{T}$, where $M$ is an orthogonal matrix with $\operatorname{det} M=-1$. Trace-type invariants, and so all the higher-spin currents, anomalies and derivatives, are inert under $\mu$. But an invariant constructed from a single $\varepsilon$ tensor changes by a factor $\operatorname{det} M=-1$ under $\mu$. Precisely this property was used in [6] to show that the conservation of the classical Pfaffian ${ }^{7}$ current $\partial_{-} \operatorname{Pf}\left(j_{+}\right)=0$, which exists iff $p=q$, generalises to the quantum theory. The anomaly must be odd under $\mu$ but even under $\tau$, which leaves $\partial_{+}\left(\varepsilon_{a_{1} b_{1} a_{2} b_{2} \ldots a_{p} b_{p}} j_{-}^{a_{1} b_{1}} j_{+}^{a_{2} b_{2}} \ldots j_{+}^{a_{p} b_{p}}\right)$ as the only possibility. Hence the $S O(2 p) / S O(p) \times S O(p)$ models contain both a quantum current of spin 4 which is even under $\mu$ and a quantum current of spin $p$ which is odd under $\mu$.

Spin-4 for complex Grassmannians

- If $p \geq q \geq 2$ then $n_{C}=2$ and $n_{B}=4$. The possible anomalies are $A_{i}$ with $1 \leq i \leq 6$ and $A_{7}$ iff $p \neq q$. Hence, $n_{A}=7$ if $p \neq q, n_{A}=6$ if $p=q$, and either way GW fails.

- If $p \geq q=1$, the target manifolds are projective spaces $C P^{p}$ with $n_{C}=1$ and $n_{B}=3$. If in addition $p \geq 2$, then $A_{2}, A_{3}$ and $A_{7}$ are independent, but $A_{4}, A_{5}$ and $A_{6}$ can be expressed in terms of them, so $n_{A}=4$ and GW fails. If $p=q=1$, however, then $A_{7}$ also reduces to a combination of $A_{2}$ and $A_{3}$, so $n_{A}=n_{B}=3$ and $\mathrm{GW}$ works; this is the case $S U(2) / U(1)=C P^{1}=S^{2}$.

We need not consider $\varepsilon$ tensors for the complex Grassmannians because the field $\Phi$ transforms under $S U(p+q)$ according to (2) a), i.e. in the tensor product of the defining representation and its conjugate. We need $\varepsilon$ tensors associated to each of these representations (one with indices up and one with indices down in traditional notation) to construct an invariant, but the product of two such tensors reduces to a combination of $\delta$ tensors, and hence to traces.

Spin-4 for quaternionic/symplectic Grassmannians

- If $p \geq q \geq 2$ then $n_{C}=2$ and $n_{B}=4$. The counting is exactly like the real case, in keeping with the remarks following (24). Hence GW works for $p=q$ but fails for $p \neq q$.

- If $p \geq q=1$ then $n_{C}=1$ and $n_{B}=3$. The anomalies behave more subtlety here. For

\footnotetext{
${ }^{7}$ The Pfaffian is defined by $\operatorname{Pf} X=c_{n} \varepsilon_{a_{1} b_{1} a_{2} b_{2} \ldots a_{n} b_{n}} X_{a_{1} b_{1}} X_{a_{2} b_{2}} \ldots X_{a_{n} b_{n}}$, for any $2 n \times 2 n$ antisymmetric matrix $X$ (real or complex), where the constant $c_{n}=\left(2^{n} n !\right)^{-1}$ ensures $(\operatorname{Pf} X)^{2}=\operatorname{det} X$.
} 
$p \geq 2$, one combination of $A_{4}, A_{5}$ and $A_{7}$ remains independent of $A_{2}$ and $A_{3}$, so $n_{A}=4$ and GW fails. But for $p=q=1$ we find $A_{4}, A_{5}$ and $A_{7}$ all reduce to $A_{2}$ and $A_{3}$, so $n_{A}=3$ and GW works; this is the case $S p(2) / S p(1) \times S p(1)=S O(5) / S O(4)=S^{4}$.

\section{Details for the remaining models}

The remaining families of classical symmetric spaces are

$$
S U(n) / S O(n), \quad S U(2 n) / S p(n), \quad S O(2 n) / U(n), \quad S p(n) / U(n)
$$

The first two and last two sequences are very similar in character.

For $S U(n) / S O(n): \Phi$ is an $n \times n$ complex matrix and (2) $3 \mathrm{~b}, 4 \mathrm{~b})$ hold with

$$
\Phi^{T}=\Phi, \quad \Phi \Phi^{*}=1, \quad \operatorname{det} \Phi=1, \quad N=1 .
$$

There is a symmetry $\mu: \Phi \mapsto M \Phi M^{T}$ where $M$ is a unitary matrix with $\operatorname{det} M=-1$. Trace-type invariants (14b), and hence all conserved quantities, anomalies and derivatives, are even under $\mu$. Any $S U(n)$-invariant constructed using an $\varepsilon$ tensor is odd under $\mu$, however, so we disregard these.

For $S U(2 n) / S p(n): \Phi$ is a $2 n \times 2 n$ complex matrix and (2b $3 \mathrm{~b}, 4 \mathrm{~b})$ hold with

$$
\Phi^{T}=-\Phi, \quad \Phi \Phi^{*}=-1, \quad \operatorname{Pf} \Phi=1, \quad N=J,
$$

where $J$ is a symplectic structure chosen to have $\operatorname{Pf} J=1$. The condition $\operatorname{Pf} \Phi=1$ implies

$$
\Phi_{\left[a_{1} b_{1}\right.} \Phi_{a_{2} b_{2}} \ldots \Phi_{\left.a_{n} b_{n}\right]}=\frac{2^{n} n !}{(2 n) !} \varepsilon_{a_{1} b_{1} a_{2} b_{2} \ldots a_{n} b_{n}}
$$

and hence any $\varepsilon$ invariant can be re-expressed in terms of traces.

In these two families of sigma models the anomalies and derivatives can be written entirely in terms of $j$ 's (because the $G$-invariants are of type (14b)), so we can restrict attention to the lists (18) and (20). We noted in section 3 that this was sufficient to show quantum conservation of the spin-2 (energy-momentum) and spin-3 currents, the latter existing when $n \geq 3$ (so that there is 3rd-order invariant [12]). For the spin-4 currents, the counting is identical for members of either family with a common value of $n$. In fact, exactly the same anomalies and derivatives occur for the spin-4 current in the $S U(n)$ PCM, to which the same lists (18) and (20) apply [11].

Spin-4 in $S U(n) / S O(n), S U(2 n) / S p(n)$ and $S U(n)(\mathrm{PCM})$

- If $n \geq 4$ then $n_{C}=2, n_{B}=4$. The anomalies are $A_{i}$ in (18): $n_{A}=5$ and GW works.

- If $n=3$ then $n_{C}=1, n_{B}=3$. One combination of $A_{4}$ and $A_{5}$ reduces to $A_{2}$ and $A_{3}$, but any distinct combination remains independent, so $n_{A}=4$ and GW fails.

- If $n=2$ then $n_{C}=1, n_{B}=3$. Now $A_{4}$ and $A_{5}$ each reduce to combinations of $A_{2}$ and $A_{3}$, so $n_{A}=3$ and GW works. These target spaces are $S U(2) / S O(2)=S^{2}$, 
$S U(4) / S p(2)=S O(6) / S O(5)=S^{5}$ and $S U(2)=S^{3}$.

(Further details relevant to the behaviour of these invariants are given in the appendix).

For $S O(2 n) / U(n): \Phi$ is a real, antisymmetric $2 n \times 2 n$ matrix and (234) hold with

$$
\Phi^{*}=-\Phi^{T}=\Phi, \quad \Phi^{2}=-1, \quad \operatorname{Pf} \Phi=1, \quad N=J,
$$

where, again, $J$ is a symplectic structure with $\operatorname{Pf} J=1$. When $n$ is even there is a symmetry $\tau: \Phi \mapsto-\Phi$; when $n$ is odd there is a symmetry $\tau: \Phi \mapsto-R \Phi R^{T}$ where $R$ is orthogonal, $\operatorname{det} R=-1$, and $R J=-J R$ (this ensures $\operatorname{Pf} \Phi=1$ is preserved); the currents (9) are unchanged by $\tau$. Once again we may disregard $\varepsilon$ invariants because (27) holds.

For $S p(n) / U(n): \Phi$ is an anti-hermitian $2 n \times 2 n$ matrix and (2) 34,4 ) hold with

$$
\Phi^{*}=-\Phi^{T}=J \Phi J^{-1}, \quad \Phi^{2}=-1, \quad \operatorname{det} \Phi=1, \quad N=J .
$$

There is a discrete symmetry $\tau: \Phi \mapsto-\Phi$ under which the higher-spin currents are inert.

For these last two families the field $\Phi$ is antihermitian and this implies (24) again (compare with the Grassmannians, for which $\Phi$ is hermitian). Consequently: (i) The trace of any odd number of currents vanishes, with or without a $\Phi$ term. (ii) $\operatorname{Tr}\left(\Phi j^{2}\right)=0$ for any $j$, from cyclicity of the trace. (iii) $\operatorname{Tr}\left(\Phi j j^{\prime}\right)$, which need not vanish if $j \neq j^{\prime}$, is even under (13) and odd under $\tau$.

Spin-4 in $S O(2 n) / U(n)$ and $S p(n) / U(n)$

- If $n \geq 4$ in the first family, or $n \geq 2$ in the second family, then $n_{C}=2$ and $n_{B}=4$. The anomalies are $A_{i}$ with $1 \leq i \leq 6, A_{7}$ being ruled out by $\tau$, so $n_{A}=6$ and GW fails.

- The remaining cases are models we have already considered:

$S O(6) / U(3)=S U(4) / S(U(3) \times U(1))=C P^{3}$ and $S O(4) / U(2)=S p(1) / U(1)=S^{2}$.

\section{The co-existence of local and non-local quantum charges}

Returning to our results, stated at the end of section 3, points (i) and (ii) imply that the integrable symmetric space models (1) always possess local (as well as non-local) quantum conserved charges. To discuss the significance of this, let us recall the situation for a PCM based on $G$. In this model the non-local charges extend the Lie algebra $\mathfrak{g}$ of $G$ to a quantum group structure, known as a Yangian $Y(\mathfrak{g}) \supset \mathfrak{g}$ (see e.g. [18, 19]) and the particle multiplets $V_{i}$ are irreducible representations (irreps) of $Y(\mathfrak{g})$ [24, 11] (actually there are 'left' and 'right' copies of this symmetry in the PCM, but this is not important here). Let us denote by $(\mu)$ the $\mathfrak{g}$-irrep with highest weight $\mu$, and let $\lambda_{i}$ be the fundamental weights dual to the simple roots and thus associated to the nodes of the $\mathfrak{g}$ Dynkin diagram. The PCM particle multiplets are $Y(\mathfrak{g})$-irreps in one-to-one correspondence with these nodes. For the $A(S U)$ and $C(S p)$ families, $V_{i}=\left(\lambda_{i}\right)$, while for the $B / D(S O)$ families the $Y(\mathfrak{g})$-irreps are not $\mathfrak{g}$-irreps in general, but they take the form $V_{i}=\left(\lambda_{i}\right) \oplus\{$ more $\}$.

One can, therefore, view the masses and 3-point couplings or fusings in the PCM Smatrices 24] as arising from properties of Yangian (non-local charge) representations and 
their tensor products. What is remarkable is that the same fusings and masses occur in affine Toda theories [14, where they follow from the existence of a set of quantum, conserved, commuting, local charges, with a particular set of spins equal to the exponents of $\mathfrak{g}$ modulo its Coxeter number $h(\mathfrak{g})$ 15. Actually, this statement requires careful interpretation in the non-simply-laced cases, since these involve a restricted fusing rule based on a dual pair of Kac-Moody algebras [16, 17. Now, it was shown in [1] that the PCMs possess classical, commuting, conserved local charges with precisely this same set of spins (see [13] for the exceptional groups) and, as described in section 3, some of them survive quantization. It is natural to conjecture [1] that all these commuting charges survive, thereby fitting very nicely with the Yangian structure.

\begin{tabular}{|c|c|c|}
\hline$G$ & exponents & $h(\mathfrak{g})$ \\
\hline$A_{n}=S U(n+1)$ & $1,2, \ldots, n$ & $n+1$ \\
$B_{n}=S O(2 n+1)$ & $1,3, \ldots, 2 n-1$ & $2 n$ \\
$C_{n}=S p(n)$ & $1,3, \ldots, 2 n-1$ & $2 n$ \\
$D_{n}=S O(2 n)$ & $1,3, \ldots, 2 n-3 ; n-1$ & $2 n-2$ \\
\hline
\end{tabular}

Exactly the same ideas can now be applied to the $G / H$ symmetric space sigma models. Here too the quantum non-local charges [1, 2] (see also [20, 21]) generate a Yangian, $Y(\mathfrak{g}) \supset$ $\mathfrak{g}$, and the $S$-matrix constructions of [3, 5] can be interpreted in these terms, although the $Y(\mathfrak{g})$ irreps differ from those in the PCMs (see below). It is also known [12] (and [13] for the exceptional cases) that the $G / H$ model possesses classical, commuting, conserved, local charges, with spins equal to the exponents of $K$, a certain Lie group/algebra which should be regarded as encoding the simple root structure of $G / H[9]$. The obvious question is whether all these commuting charges could survive quantization, and in particular whether this would be consistent with the $S$-matrices proposed in [3] and [5].

\begin{tabular}{|c|c|c|}
\hline$G / H$ & $K$ & $V_{i}$ \\
\hline$S^{n}=S O(n+1) / S O(n)$ & $A_{1}$ & $\left(\lambda_{1}\right)$ \\
$S U(2 n+2) / S p(n+1)$ & $A_{n}=S U(n+1)$ & $\left(\lambda_{2 i}\right)$ \\
$S p(2 n) / S p(n) \times S p(n)$ & $C_{n}=S p(n)$ & $\left(\lambda_{2 i}\right)$ \\
$S U(n+1) / S O(n+1)$ & $A_{n}=S U(n+1)$ & $\left(2 \lambda_{i}\right)$ \\
$S O(2 n) / S O(n) \times S O(n)$ & $D_{n}=S O(2 n)$ & $\left(2 \lambda_{i}\right) \oplus\{$ more $\}$ \\
\hline
\end{tabular}

For a basic test of this suggestion, consider the more detailed conclusions (iii), (iv) and also $(\mathrm{v})$, at the end of section 3 . Noting that a conserved charge of spin $s$ corresponds to a conserved current of spin $s+1$, we see that, for those models which are actually quantum 
integrable, a classical conservation law with $s=2$ always survives. We have shown that conservation laws with $s=3$ also survive quantization in these theories, with the possible exception of the $S U(3) \mathrm{PCM}$ and the $S U(3) / S O(3)$ and $S U(6) / S p(3)$ sigma-models, for which GW fails. But these are exactly the integrable examples for which there is no charge of spin 3 in the classically commuting set constructed in [11, 12, as can be checked from the data in the tables. Thus the GW analysis is precisely compatible with the claim that the classical commuting charges generalise to the quantum theory.

Much more stringent consistency requirements follow by taking the fusings that would be predicted by the quantum local charges, according to Dorey's rule [15], and comparing these with the behaviour of the Yangian representations $V_{i}$ given in the table. We have included the $S^{n}$ models for completeness; these have just a single irrep which is the vector of $\mathfrak{s o}(n+1)$ [25. For the remaining families, the representations $V_{i}$ with $i=1, \ldots, n$ are those proposed in the S-matrix constructions of [3, 5]. Note that $\left(2 \lambda_{i}\right)$ appears in the symmetrized product of the $\left(\lambda_{i}\right)$ with itself. The $S O(2 n) / S O(n) \times S O(n)$ case is by far the most complicated, because in general the $Y(\mathfrak{s o}(2 n))$-irreps are not $\mathfrak{s o}(2 n)$-irreps. However, those built from the vector $(i=1)$ and spinor $(i=n-1, n)$ representations are: $V_{i}=\left(2 \lambda_{i}\right)$, for $i=1, n-1, n .^{8}$

For three of the families it is relatively straightforward to compute the three-pointcouplings. For $S U(2 n+2) / S p(n+1)$ and $S p(2 n) / S p(n) \times S p(n)$ the multiplets are just a subset of the fundamental $Y(\mathfrak{g})$-irreps (the even-labelled ones), which are also $\mathfrak{g}$-irreps, and so their tensor products and associated S-matrix structure is fully understood. It is clear from [5] and references therein that the fusings are indeed those of the $A_{n}$ and restricted $C_{n}$ cases respectively, as would be expected from Dorey's rule [15, 17]. In the latter case, however, the masses are those of the $A_{2 n}^{(2)}$ Toda theory [27] (for which the $R$-matrix fusings are the same as $C_{n}$ ); it would be interesting to investigate this case in more detail (similar subtleties arise in comparing the non-simply-laced Toda models and PCMs [11]). For $S U(n+1) / S O(n+1)$ the irreps $V_{i}$ are still $\mathfrak{g}$-irreps, but they are not fundamental, so some work must be done. Using the tensor product graph (TPG) [28, one can show that $V_{j}=\left(2 \lambda_{j}\right)$ and $V_{k}=\left(2 \lambda_{k}\right)$ fuse to give, for example, $V_{j+k}=\left(2 \lambda_{j+k}\right)$ at $\theta=i \pi(j+k) /(n+1)$ if $j+k<n+1$. In general we find exactly the same fusings and rapidity differences as for the irreps $\left(\lambda_{j}\right)$ and $\left(\lambda_{k}\right)$ with the original, undoubled, weights. The couplings and masses are therefore those of $A_{n}$, in accordance, once again, with expectations based on quantum local charges.

The $S O(2 n) / S O(n) \times S O(n)$ case is, of course, harder, and we are unable to carry out all the necessary calculations because the TPG cannot handle $\mathfrak{g}$-reducible representations. However, one can compute the TPGs for the irreps $V_{1}, V_{n-1}$ and $V_{n}$ with one another $\left(V_{1} \otimes V_{1}\right.$ is given in [3, 28]) and it is remarkable that once again the same couplings occur,

${ }^{8}$ The first few higher tensor multiplets after $V_{1}$ are $V_{2} \equiv\left(2 \lambda_{2}\right) \oplus\left(\lambda_{2}\right) \oplus(0),\left(2 \lambda_{3}\right) \oplus\left(\lambda_{3}+\lambda_{1}\right) \oplus V_{1}$, $\left(2 \lambda_{4}\right) \oplus\left(\lambda_{4}+\lambda_{2}\right) \oplus\left(\lambda_{4}\right) \oplus V_{2}$; the full formula is given in [26]. 
and at the same rapidity differences, as for $\left(\lambda_{1}\right),\left(\lambda_{n-1}\right)$ and $\left(\lambda_{n}\right)$ in $D_{n}$. It is natural to expect, therefore, that the same applies for all the $V_{i}$ and that the masses and couplings are just those of $D_{n}$.

Given our findings, it would be interesting to investigate whether the central result of [17] - on the Dorey's-rule structure of tensor products of fundamental $Y(\mathfrak{g})$ representationsholds more generally when the highest weights of the representations are doubled, or perhaps even multiplied by some integer. Another very natural question is whether there is any deeper significance to the root system $K$ from which one could gain a more direct understanding of the multiplet structure in the quantum $G / H$ model.

Acknowledgments: The research of JME is supported in part by Gonville and Caius College, Cambridge. CASY is supported by a PPARC studentship. DK is grateful for a NSF graduate research fellowship and a Columbia University Euretta J. Kellett fellowship. This work was also supported by the EU 'EUCLID' network, ref. HPRN-CT-2002-00325.

\section{Appendix: more on certain 4th-order invariants}

To apply the GW counting argument, we need to know which of the anomaly terms $A_{i}$ with $2 \leq i \leq 7$ in (18) and (19) are independent when there is no primitive 4th-order invariant.

- $S U(n), S U(n) / S O(n)$ and $S U(2 n) / S p(n)$ : These spaces have a primitive symmetric 4th-order invariant when $n \geq 4\left[12\right.$ and the anomalies $A_{i}$ with $2 \leq i \leq 5$ in (18) are then independent. When $n<4$ there is no primitive 4 th-order invariant and so one combination of $A_{4}$ and $A_{5}$ can be expressed in terms of $A_{2}$ and $A_{3}$. To see whether or not there is still one combination of $A_{4}$ and $A_{5}$ which is independent of $A_{2}$ and $A_{3}$ it suffices, by linearity, to determine whether or not

$$
\operatorname{Tr}\left(X^{2} Y^{2}\right), \quad \operatorname{Tr}\left(X^{2}\right) \operatorname{Tr}\left(Y^{2}\right), \quad(\operatorname{Tr} X Y)^{2}
$$

are independent for $X, Y \in \mathfrak{m}$ (where, for $S U(n), \mathfrak{m}=\mathfrak{s u}(n)$, its Lie algebra).

It is easy to check that $\operatorname{Tr} X^{4}=\frac{1}{2}\left(\operatorname{Tr} X^{2}\right)^{2}$ for $X \in \mathfrak{m}=\mathfrak{s u}(2)$ or $\mathfrak{s u}(3)$ and that $\operatorname{Tr}\left(X^{2} Y^{2}\right)=\frac{1}{2}\left(\operatorname{Tr} X^{2}\right)\left(\operatorname{Tr} Y^{2}\right)$ for $X, Y \in \mathfrak{s u}(2)$ ( or, in a different representation, $\operatorname{Tr}\left(X^{2} Y^{2}\right)=$ $\frac{1}{4}\left(\operatorname{Tr} X^{2}\right)\left(\operatorname{Tr} Y^{2}\right)+\frac{1}{4}(\operatorname{Tr} X Y)^{2}$ for $\left.X, Y \in \mathfrak{s o}(3)\right)$. But the quantities (30) are independent for $X, Y \in \mathfrak{s u}(3)$, as may be checked by judicious choices of these matrices.

The symmetric spaces $S U(n) / S O(n)$ and $S U(2 n) / S p(n)$ behave very similarly. For the first family, $\mathfrak{m} \subset \mathfrak{s u}(n)$, consisting of symmetric, imaginary matrices. For the second fam-

ily, $\mathfrak{m}$ consists of matrices of the block form $\left(\begin{array}{cc}x & y \\ y^{*} & -x^{*}\end{array}\right)$ with $x \in \mathfrak{s u}(n)$ and $y$ complex and $n \times n$ with $y^{T}=-y$. In both cases the quantities (30) are easily seen to be independent for $n=3$, but for $n=2$ standard properties of $s u(2)$ matrices imply that $X^{2}$ and $Y^{2}$ are multiples of the identity matrix and hence the first two expressions in (30) are proportional. 
- Grassmannians: For $X, Y \in \mathfrak{m}$ we have the block forms

$$
X=\left(\begin{array}{cc}
0 & x \\
-x^{\dagger} & 0
\end{array}\right), \quad Y=\left(\begin{array}{cc}
0 & y \\
-y^{\dagger} & 0
\end{array}\right), \quad N=\left(\begin{array}{cc}
1 & 0 \\
0 & -1
\end{array}\right),
$$

where $x, y$ are $p \times q$ real or complex matrices, or in the quaternionic case $2 p \times 2 q$ complex matrices obeying a symplectic reality condition. Using the fact that $g^{-1} \Phi g=N$ with $g^{-1} j g \in \mathfrak{m}$ for each current $j$, any relations among $A_{i}$ with $2 \leq i \leq 7$ in (18) and (19) are equivalent to relations among:

$$
\begin{aligned}
\operatorname{Tr} X^{2} \operatorname{Tr} Y^{2} & =4 \operatorname{Tr} x^{\dagger} x \operatorname{Tr} y^{\dagger} y \\
\operatorname{Tr} X Y \operatorname{Tr} X Y & =\left(\operatorname{Tr} x^{\dagger} y+\operatorname{Tr} y^{\dagger} x\right)^{2} \\
\operatorname{Tr} N X Y \operatorname{Tr} N X Y & =\left(\operatorname{Tr} x^{\dagger} y-\operatorname{Tr} y^{\dagger} x\right)^{2} \\
\operatorname{Tr} X Y X Y & =\operatorname{Tr} x y^{\dagger} x y^{\dagger}+\operatorname{Tr} x^{\dagger} y x^{\dagger} y \\
\operatorname{Tr} X^{2} Y^{2} & =\operatorname{Tr} x x^{\dagger} y y^{\dagger}+\operatorname{Tr} x^{\dagger} x y^{\dagger} y \\
\operatorname{Tr}\left(N X^{2} Y^{2}\right) & =\operatorname{Tr} x x^{\dagger} y y^{\dagger}-\operatorname{Tr} x^{\dagger} x y^{\dagger} y
\end{aligned}
$$

These formulas can be used to check various statements made in section 5. In particular, in the real and complex cases with $q=1, x$ and $y$ are $p$-component column vectors. In the real case, with $p \geq 2$, there are clearly 2 invariants $x^{2} y^{2}$ and $\left(x^{T} y\right)^{2}$. In the complex case there are 3 independent combinations if $p \geq 2$, namely $\left(x^{\dagger} x\right)\left(y^{\dagger} y\right),\left(x^{\dagger} y\right)\left(y^{\dagger} x\right)$ and $\left(x^{\dagger} y+y^{\dagger} x\right)^{2}$; but these reduce to 2 if $p=1$, namely $\left(x^{*} x\right)\left(y^{*} y\right)$ and $\left(x^{*} y+y^{*} x\right)^{2}$. In the quaternionic case $x$ and $y$ are $2 p \times 2$ matrices, even when $q=1$. The independent combinations are $\left(\operatorname{Tr} x^{\dagger} x\right)\left(\operatorname{Tr} y^{\dagger} y\right),\left(\operatorname{Tr} x^{\dagger} y\right)\left(\operatorname{Tr} y^{\dagger} x\right)$ and a third, $\operatorname{Tr}\left(x^{\dagger} y x^{\dagger} y\right)=\operatorname{Tr}\left(y^{\dagger} x y^{\dagger} x\right)$ which is independent if $p \geq 2$, but not if $p=1$.

\section{References}

[1] M. Lüscher, Quantum nonlocal charges and absence of particle production in the two-dimensional nonlinear sigma model, Nucl. Phys. B135 (1978) 1.

[2] E. Abdalla, M. Forger and M. Gomes, On the origin of anomalies in the quantum nonlocal charge for the generalized nonlinear sigma models, Nucl. Phys. B210 (1982) 181.

[3] P. Fendley, Integrable sigma models with $\theta=\pi$, Phys. Rev. B 63 (2001) 104429 arXiv:cond-mat/0008372.

[4] P. Fendley, Integrable sigma models and perturbed coset models, JHEP 0105 (2001) 050 arXiv:hep-th/0101034.

[5] A. Babichenko, Quantum integrability of sigma models on AII and CII symmetric spaces, Phys. Lett. B554 (2003) 96 arXiv:hep-th/0211114.

[6] J.M. Evans, D. Kagan and C.A.S. Young, Non-local charges and the quantum integrability of sigma models on the symmetric spaces $S O(2 n) / S O(n) \times S O(n)$ and $S p(2 n) / S p(n) \times S p(n)$, Phys. Lett. B597 (2004) 112 arXiv:hep-th/0404003. 
[7] S. Parke, Absence of particle production and factorization of the $S$ matrix in (1+1)-dimensional models, Nucl. Phys. B174 (1980) 166.

[8] Y.Y. Goldschmidt and E. Witten, Conservation laws in some two-dimensional models, Phys. Lett. B91 (1980) 392.

[9] S. Helgason, Differential geometry, Lie groups, and symmetric spaces, Academic Press, New York, 1978.

[10] H. Eichenherr and M. Forger, Higher local conservation laws for nonlinear sigma models on symmetric spaces, Commun. Math. Phys. 82 (1981) 227.

[11] J.M. Evans, M. Hassan, N.J. MacKay and A.J. Mountain, Local conserved charges in principal chiral models, Nucl. Phys. B561 (1999) 385 arXiv:hep-th/9902008;

[12] J.M. Evans and A.J. Mountain, Commuting charges and symmetric spaces, Phys. Lett. B483 (2000) 290 arXiv:hep-th/0003264.

[13] J.M. Evans, Integrable sigma-models and Drinfeld-Sokolov hierarchies, Nucl. Phys. B608 (2001) 591 arXiv:hep-th/0101231.

[14] E. Corrigan, Recent developments in affine Toda field theory, Lectures at the CRM-CAP Summer School Particles and Fields 94 (Banff, Canada) arXiv:hep-th/9412213.

[15] P.E. Dorey, Root systems and purely elastic S-matrices, Nucl. Phys. B358 (1991) 654.

[16] P.E. Dorey, A remark on the coupling dependence in Affine Toda field theories, Phys. Lett. B312 (1993) 291.

[17] V. Chari and A. Pressley, Yangians, integrable quantum systems and Dorey's rule, Commun. Math. Phys. 181 (1996) 265 arXiv:q-alg/9505085.

[18] D. Bernard, Hidden Yangians in 2D massive current algebras, Commun. Math. Phys. 137 (1991) 191.

[19] N.J. MacKay, On the classical origins of Yangian symmetry in integrable field theory, Phys. Lett. B281 (1992) 90, Erratum-ibid. B308 (1993) 444.

[20] H. Eichenherr and M. Forger, On the dual symmetry of the non-linear sigma models, Nucl. Phys. B155 (1978) 381; More about non-linear sigma models on symmetric spaces, Nucl. Phys. B164 (1980) 528, Erratum-ibid. B282 (1987) 745.

[21] E. Abdalla, M.C.B. Abdalla, J.C. Brunelli, A. Zadra, The algebra of non-local charges in non-linear sigma models, Comm. Math. Phys. 166 (1994) 379.

[22] J-P. Antoine and B. Piette, Classical nonlinear $\sigma$ models on Grassmann manifolds of compact and noncompact type, J. Math. Phys. 28 (1987) 2753.

[23] N. MacKay and B. Short, Boundary scattering, symmetric spaces and the principal chiral model on the half-line, Comm. Math. Phys. 233 (2003) 313 arXiv:hep-th/0104212.

[24] E. Ogievetsky, N. Reshetikhin and P. Wiegmann, Principal chiral field in two dimensions on classical Lie algebras: the Bethe ansatz solution and classical theory of scattering, Nucl. Phys. B280 (1987) 45. 
[25] A.B. Zamolodchikov and Al.B. Zamolodchikov, Factorized S-matrices in two dimensions as the exact solutions of certain relativistic quantum field theory models, Ann. Phys. 120 (1979) 253.

[26] M. Kleber, Combinatorial structure of finite-dimensional representations of Yangians: the simply-laced case, Int. Math. Res. Notices 4 (1997) 187 arXiv:q-alg/9611032.

[27] G.W. Delius, M.D. Gould and Y-Z. Zhang, Twisted quantum affine algebras and solutions to the Yang-Baxter equation, Int. J. Mod. Phys. A11 (1996) 3415 arXiv:q-alg/9508012.

[28] N.J. MacKay, Rational R-matrices in irreducible representations, J. Phys. A24 (1991) 4017; R-B. Zhang, M.D. Gould and A.J. Bracken, From representations of the braid group to solutions of the Yang-Baxter equation, Nucl. Phys. B354 (1991) 625. 